\title{
Curves of genus 2 and Desargues configurations
}

\author{
D. Avritzer* H. Lange*
}

\section{Introduction}

A Desargues configuration is the configuration of 10 points and 10 lines of the classical theorem of Desargues in the complex projective plane. For a precise definition see Section 2. The greek mathematician C. Stephanos showed in 1883 (see [11]) that one can associate to every Desargues configuration a curve of genus 2 in a canonical way. Moreover he proved that the induced map from the moduli space of Desargues configurations to the moduli space of curves of genus 2 is birational. Our main motivation for writing this paper was to understand the last result. Stephanous needed about a hundred pages of classical invariant theory to prove it. We apply instead a simple argument of Schubert calculus to prove a slightly more precise version of his result.

Let $M_{D}$ denote the (coarse) moduli space of Desargues configurations. It is a threedimensional quasiprojective variety. On the other hand, let $\mathcal{M}_{6}^{b}$ denote the moduli space of stable binary sextics. There is a canonical isomorphism $\overline{\mathcal{H}}_{g} \backslash \Delta_{1} \cong \mathcal{M}_{6}^{b}$ (see [1]). Here $\overline{\mathcal{H}}_{g}$ denotes the moduli space of stable curves of genus 2 in the sense of Deligne-Mumford and $\Delta_{1}$ the boundary divisor parametrizing 2 curves of genus 1 intersecting transversely in one point. So instead of curves of genus 2 , we may speak of binary sextics. The main result of the paper consists of the following 2 statements:

(1) There is a canonical injective birational morphism

$$
\Phi: M_{D} \hookrightarrow \mathcal{M}_{6}^{b}
$$

(2) We determine a hypersurface $H \subset \mathcal{M}_{6}^{b}$ such that

$$
\mathcal{M}_{6}^{b} \backslash H \subseteq \Im m(\Phi) \subseteq \mathcal{M}_{6}^{b}
$$

where both the inclusions above are strict.

A Desargues configuration is called special if one of its lines contains 4 configuration points. We show:

(3) A Desargues configuration $D$ is special if and only if the binary sextic $\Phi(D)$ admits a double point, i.e. the corresponding curve of genus 2 is not smooth.

*Both authors would like to thank the GMD (Germany) and CNPq (Brasil) for support during the preparation of this paper 
Figure 1: Desargues' Theorem

The Moduli space $M_{D}$ admits a natural compactification $\bar{M}_{D}$. We also study the configurations corresponding to boundary points $\bar{M}_{D} \backslash M_{D}$, which we call degenerate Desargues configurations. In fact, there are degenerate Desargues configurations of the first, second and third kind (see Section 2). As a rational map of normal projective varieties $\Phi: \bar{M}_{D} \cdots \longrightarrow \overline{\mathcal{M}}_{6}^{b}$ is defined in codimension 1 . Hence it extends to a morphism on an open set of the divisor $\bar{M}_{D} \backslash M_{D}$. We show, however:

(4) The geometric interpretation of the morphism $\Phi: \bar{M}_{D} \cdots \longrightarrow \overline{\mathcal{M}}_{6}^{b}$ does not extend to an open set $U: M_{D} \subset U \subset \bar{M}_{D}$, where the first inclusion is strict. In fact, the binary sextic associated to a degenerate Desargues configuration of the first kind in an analogous way is not semistable.

In Section 2, we construct the moduli spaces $M_{D}$ and its compactification $\bar{M}_{D}$. In Section 3, we give the definition of the map $\Phi: M_{D} \longrightarrow \mathcal{M}_{6}^{b}$. Sections 4, 5, 6 and 7 contain the proofs of statements (1), (2), (3) and (4) respectively.

The second author would like to thank W. Barth with whom he discussed the subject, already 15 years ago.

\section{The moduli space of Desargues configurations}

Let $\mathbb{P}_{2}$ denote the projective plane over the field of complex numbers. The classical theorem of Desargues says: If the lines joining corresponding vertices of two triangles $A_{1}, B_{1}, C_{1}$ and $A_{2}, B_{2}, C_{2}$ in $\mathbb{P}_{2}$ meet in a point $A$ then the intersections of corresponding sides lie on a line $a$ and conversely (see Figure 1 ).

The triangles are then said to be in perspective, $A$ is called the center of perspective and $a$ the axis of perspective. The configuration consisting of the 10 points $A, A_{i}, B_{i}, C_{i},(i=$ $1,2,3)$ and ten lines, namely the 6 sides of the triangles, the 3 lines joining $A$ to the vertices of the triangle and the axis of perspective, is called a Desargues configuration. It is a $10_{3}$ - configuration meaning that each of the 10 lines contains 3 of the 10 points and through each of the 10 points there pass 3 of the 10 lines.

It may happen that one of the vertices of one triangle lies on the opposite side of the other triangle, in which case Desargues Theorem is still valid, but one line contains now 4 of the 10 points. Contrary to some authors, we consider this configuration also as a Desargues configuration and call it a special Desargues configuration.

As long ago as 1846, Cayley remarked (see 迎, p.318) that the 10 lines and 10 planes determined by 5 points $e_{1}, e_{2}, e_{3}, e_{4}, e_{5}$ in general position in $\mathbb{P}_{3}$ meet a plane $\pi$ not containing any of the points $e_{i}$ in a Desargues configuration. Conversely, it follows from the standard proof of Desargues theorem via $\mathbb{P}_{3}$ (see [12]) that every Desargues configuration is obtained in this way. In the sequel, we choose the coordinates of $\mathbb{P}_{3}$ in such a way that

$$
e_{1}=(1: 0: 0: 0), \ldots, e_{4}=(0: 0: 0: 1), e_{5}=(1: 1: 1: 1)
$$


Figure 2: Desargues' Theorem in space

If the plane $\pi$ is given by the equation $\sum_{i=1}^{4} \alpha_{i} x_{i}=0$, then the assumption that $e_{i} \notin \pi$ for $i=1, \ldots, 5$ amounts to

$$
\alpha_{i} \neq 0 \text { for } i=1, \ldots, 4 \text { and } \sum_{i=1}^{4} \alpha_{i} \neq 0 .
$$

We denote by $D_{\pi}$ the Desargues configuration determined by $\pi$. It consists of the 10 points $p_{i j}:=\overline{e_{i} e_{j}} \cap \pi$ and the 10 lines $\ell_{i j k}:=\overline{e_{i} e_{j} e_{k}} \cap \pi$ for $i \leq i, j, k \leq 5, i \neq j \neq k \neq i$ (see Figure 2). Note that the notation is meant to be symmetric, i.e. $p_{i j}=p_{j i}$ and $\ell_{i j k}=\ell_{j k i}$, etc. From the picture it is obvious that every point of a Desargues configuration is the center of perspective of two triangles: the point $p_{i j}$ is the centre of perspective of the triangles $p_{i k} p_{i l} p_{i m}$ and $p_{j k} p_{j l} p_{j m}$ where $\{i, j, k, l, m\}=\{1,2,3,4,5\}$. In particular every point of the configuration admits an axis of perspective: for $p_{i j}$ it is the line $\ell_{k l m}$.

By definition two Desargues configurations $D_{1}$ and $D_{2}$ are isomorphic if there is an automorphism $\alpha \in P G L_{2}(\mathbb{C})$ such that $D_{2}=\alpha\left(D_{1}\right)$.

Lemma 2.1. For planes $\pi$ and $\pi^{\prime} \in \mathbb{P}_{3}$ not containing a point $e_{i}$ the following conditions are equivalent:

1) $D_{\pi}$ is isomorphic to $D_{\pi^{\prime}}$.

2) There is an $A \in P G L_{3}(\mathbb{C})$ such that

a) $A \pi=\pi^{\prime}$

b) A permutes the 5 points $e_{1}, \ldots, e_{5}$.

For the proof we need the following notion: a complete quadrangle of a Desargues configuration $D$ is a set of 4 points and six lines of $D$ which form the points and lines of a complete quadrangle. From Figure 2 it is obvious that $D_{\pi}$ (and thus any $D$ ) admits exactly 5 complete quadrangles: any $e_{i}$ determines the complete quadrangle consisting of the points $p_{i j}, p_{i k}, p_{i l}, p_{i m}$ and the lines $\ell_{i j k}, \ell_{i j l}, \ell_{i j m}, \ell_{i k l}, \ell_{i k m}, \ell_{i l m}$.

Proof of Lemma 1.1: We have to show that (1) implies (2), the converse implication being obvious. So let $\alpha: \pi \longrightarrow \pi^{\prime}$ be a linear isomorphism with $\alpha\left(D_{\pi}\right)=D_{\pi^{\prime}}$. We have to show that $\alpha$ extends in a unique way to an $A \in P G L_{3}(\mathbb{C})$ permuting the 5 points $e_{1}, \ldots, e_{5}$. Certainly $\alpha$ maps the 5 complete quadrangles of $D_{\pi}$ onto the 5 complete quadrangles of $D_{\pi^{\prime}}$. As outlined above a complete quadrangle of $D_{\pi}$ is uniquely determined by a point $e_{i}$ and similarly for $D_{\pi^{\prime}}$. Hence $\alpha$ induces a permutation $\sigma$ of the 5 points $e_{1}, \ldots, e_{5}$. It is easy to see that there is a one-dimensional family $\left\{A_{t} \in P G L_{3}(\mathbb{C}) \mid t \in \mathbb{P}_{1}\right\}$ satisfying $A_{t}\left(e_{1}\right)=e_{\sigma(1)}$ and $A_{t}(\pi)=\pi^{\prime}$ (choose suitable coordinates for the source$\mathbb{P}_{3}$ and the image- $\mathbb{P}_{3}$ of $\left.A: \mathbb{P}_{3} \longrightarrow \mathbb{P}_{3}\right)$. For every $t \in \mathbb{P}_{1}$ the automorphism $A_{t}$ maps the line $\overline{e_{1} p_{12}}$ onto the line $\overline{e_{\sigma(1)} p_{\sigma(1) \sigma(2)}}$. Since $e_{2} \in \overline{e_{1} p_{12}}, e_{\sigma(2)} \in \overline{e_{\sigma(1)} p_{\sigma(1) \sigma(2)}}$, and $\left\{A_{t}\left(e_{2}\right) \mid t \in \mathbb{P}_{1}\right\}=\overline{e_{\sigma(1)} p_{\sigma(1) \sigma(2)}}$, there is a unique $t_{0} \in \mathbb{P}_{1}$ such that

$$
A_{t_{0}}=e_{\sigma(2)}
$$


We claim that $A:=A_{t_{0}}$ satisfies apart from $(a)$ also condition $(b)$. But for $3 \leq k \leq 5$ we have $e_{k}=\overline{e_{1} p_{1 k}} \cap \overline{e_{2} p_{2 k}}$. Hence

$$
A\left(e_{k}\right)=A\left(\overline{e_{1} p_{1 k}}\right) \cap A\left(\overline{e_{2} p_{2 k}}\right)=\overline{e_{\sigma(1)} p_{\sigma(1) \sigma(k)}} \cap \overline{e_{\sigma(2)} p_{\sigma_{2} \sigma(k)}}=e_{\sigma(k)}
$$

This concludes the proof of the Lemma.

The 5 points $e_{1}, \ldots, e_{5}$ form a projective basis of $\mathbb{P}_{3}$. Hence for every permutation $\sigma$ of the points $e_{1}, \ldots, e_{5}$ there is a unique $A_{\sigma} \in P G L_{3}(\mathbb{C})$ inducing $\sigma$. Let $\mathcal{S}_{5}$ denote the group of these $A_{\sigma}$. The action of $\mathcal{S}_{5}$ on $\mathbb{P}_{3}$ induces an action on the dual projective space $\mathbb{P}_{3}^{*}$. Define

$$
U:=\mathbb{P}_{3}^{*} \backslash \cup_{i=1}^{5} P_{e_{i}}
$$

where $P_{e_{i}}$ denotes the plane in $\mathbb{P}_{3}^{*}$ parametrizing the planes $\pi$ with $e_{i} \in \pi$. The action of $\mathcal{S}_{5}$ on $\mathbb{P}_{3}^{*}$ restricts to an action on $U$. Since the quotient of a quasi-projective variety by a finite group is always an algebraic variety, we obtain as an immediate consequence of Lemma 2.1

Theorem 2.2. The algebraic variety $M_{D}:=U / \mathcal{S}_{5}$ is a moduli space for Desargues configurations.

It is clear how to define families of Desargues configurations. Doing this it is easy to see that $M_{D}$ is a coarse moduli space in the sense of geometric invariant theory. In particular, $M_{D}$ is uniquely determined as an algebraic variety. Since a Desargues configuration may admit a nontrivial group of automorphisms, $M_{D}$ is not a fine moduli space.

Next we work out the subspace of $M_{D}$ parametrizing special Desargues configurations. By definition a Desargues configuration $D$ is called special if and only if $D$ contains a point lying on its axis. The axis of a point $p_{i j}$ is the line $\ell_{k l m}$ with $\{i, j, k, l, m\}=\{1,2,3,4,5\}$. This implies that a configuration $D_{\pi}$ is special if and only if the plane contains the point of intersection of a line $\overline{e_{i} e_{j}}$ with the plane $\overline{e_{k} e_{l} e_{m}}$. Hence a Desargues configuration $D_{\pi}$ is special if and only if the plane $\pi$ contains a point $Q_{i j}=\overline{e_{i} e_{j}} \cap \overline{e_{k} e_{l} e_{m}},\{i, j, k, l, m\}=$ $\{1, \ldots, 5\}$. Now let $P_{i j} \subset U=\mathbb{P}_{3}^{*} \backslash \cup_{i=1}^{5} P_{e_{i}}$, denote the hyperplane parametrizing the planes $\pi$ in $\mathbb{P}_{3}$ not containing the points $e_{i}$, but containing the point $Q_{i j}$. If $q: U \longrightarrow M_{D}$ denotes the natural projection map, then we conclude, since the group $\mathcal{S}_{5}$ obviously acts transitively on the set of points $\left\{Q_{i j}\right\}$ :

Proposition 2.3. The special Desargues configurations are parametrized by the irreducible divisor $q\left(P_{12}\right)$ in $M_{D}$.

In other words: if $D$ is a special Desargues configuration then there is a plane $\pi \subset \mathbb{P}_{3}$ containing the point $P_{12}=(1: 1: 0: 0)$ and not containing any $e_{i}$ such that $D_{\pi} \cong D$.

Finally we introduce degenerate Desargues configurations. Desargues' Theorem fails if one of the two triangles is replaced by three lines passing through the center of perspective. 
Figure 3: A degenerate Desargues configuration of the 1st kind

However, the following classical theorem (see [9], p.99, Exercise 14) may be considered as a limiting case of Desargues' Theorem: If $A B C$ is a proper triangle and $a, b, c$ are 3 lines passing through a point $O$ (not lying on the sides of the triangle) then the points $a \cap \overline{B C}, b \cap \overline{A C}, c \cap \overline{A B}$ are collinear if and only if there is a an involution $\imath$ of the $\mathbb{P}_{1}$ of lines centered in $O$ such that $\imath(a)=\overline{O A}, \imath(b)=\overline{O B}, \imath(C)=\overline{O C}$.

It is now easy to see that for any configuration of 7 points and 10 lines in $\mathbb{P}_{2}$ satisfying the conditions of the theorem there is a plane $\pi \subset \mathbb{P}_{3}$ containing exactly 1 of the 5 points $e_{i}$ such that the configuration is isomorphic to the configuration $D$ cut out on $\pi$ by the lines $\overline{e_{i} e_{j}}$ and the planes $\overline{e_{i} e_{j} e_{k}}$. A picture of a degenerate Desargues configuration $D_{\pi}$ is shown in Figure 3 where $e_{5} \in \pi$ and hence $p_{15}=p_{25}=p_{35}=p_{45}=e_{5}$ with the notation above.

Note that of the 5 complete quadrangles of the Desargues configuration 4 still survive in the degenerate case namely (in the case of Figure 3) $\left\{p_{12}, p_{13}, p_{14}, p_{15}\right\},\left\{p_{12}, p_{23}, p_{24}, p_{25}\right\}$, $\left\{p_{13} . p_{23}, p_{34}, p_{35}\right\},\left\{p_{14}, p_{24}, p_{34}, p_{45}\right\}$. This implies that the proof of Lemma 1.1 also works in this case. In other words: two degenerate Desargues configurations $D_{\pi}$ and $D_{\pi^{\prime}}$ are isomorphic if and only if there is an automorphism $A \in P G L_{3}(\mathbb{C})$ with $A \pi=\pi^{\prime}$ permuting the 5 points $e_{1}, \ldots, e_{5}$ in $\mathbb{P}_{3}$. Similar remarks can be made if the configuration is even more degenerate, that is if 2 triangles of a Desargues configuration collapse (equivalently if the plane $\pi$ passes through 2 of the points $e_{i}$ (see Figure 4 where the triangle $p_{14} p_{24} p_{34}$ of Figure 3 collapsed) or if 2 triangles collapse and the 2 points come together (equivalently if the plane $\pi$ passes through 3 of the points $e_{i}$ ). In this case the configuration is a complete quadrangle (see Figure 5 , where $e_{3}=p_{13}=p_{23}$ ). We omit the details since they are easy to work out (see also Section 6).

These remarks induce the following definitions: as above fix 5 points $e_{1}, \ldots, e_{5} \in \mathbb{P}_{3}$ in general position and consider any plane $\pi \subset \mathbb{P}_{3}$. The 10 lines $\overline{e_{i} e_{j}}$ and 10 planes $\overline{e_{i} e_{j} e_{k}}$ in $\mathbb{P}_{3}$ cut out on $\pi$ a configuration $D_{\pi}$ of points and lines. We call $D_{\pi}$ a generalized Desargues configuration. If $\pi$ contains a point $e_{i}, D_{\pi}$ is called a degenerate Desargues configuration. It is called of the $i$-th kind if $\pi$ contains exactly $i$ of the points $e_{i}$ for $i=1,2,3$.

It is easy to see that Lemma 1.1 remains valid for generalized Desargues configurations. In fact the same proof works also in the degenerate case. One has only to remark that a degenerate Desargues configuration $D_{\pi}$ of the first kind (respectively $2^{\text {nd }}$ kind, respectively $3^{\text {rd }}$ kind) admits 4 (respectively 3 , respectively 1 ) complete quadrangles. In the same way as we deduced Theorem 1.2 from Lemma 1.1 we obtain from this

Theorem 2.4. The variety $\bar{M}_{D}:=\mathbb{P}_{3}^{*} / \mathcal{S}_{5}$ is a moduli space for generalized Desargues configurations.

Remark 2.5. In [5] which is the standard reference for Desargues configurations, K. Mayer constructs the moduli space $M_{D}$ in a different way. He chooses the 4 points of a complete quadrangle as a projective basis of $\mathbb{P}_{2}$, say $A, A_{1}, B_{1}, C_{1}$ in Figure 1 . Consider the 
Figure 4: A degenerate Desargues configuration of the 2nd kind

points $A_{1}^{\prime}:=\overline{A A_{1}} \cap \overline{B_{1} C_{1}}, B_{1}^{\prime}:=\overline{A B_{1}} \cap \overline{A_{1} C_{1}}$ and $C_{1}^{\prime}=\overline{A C_{1}} \cap \overline{A_{1} B_{1}}$. The three crossratios $\left(A, A_{1}, A_{1}^{\prime}, A_{2}\right),\left(A, B_{1}, B_{1}^{\prime}, B_{2}\right)$ and $\left(A, C_{1}, C_{1}^{\prime}, C_{2}\right)$ determine the Desargues configuration and an open set $V$ of $\mathbb{P}_{1}^{3}$ represents Desargues configurations. The choice of a complete quadrangle induces an action of $\mathcal{S}_{5}$ on $V$ which Mayer worked out explicitly. Moreover he showed that $V / \mathcal{S}_{5}$ is the moduli space of Desargues configurations. For our purposes, the construction of Theorem 1.2 is more appropriate.

\section{The map $\Phi: M_{D} \longrightarrow \mathcal{M}_{6}^{b}$}

Recall that a binary form $f(x, y)$ of degree 6 is called stable if $\mathrm{f}$ admits no root of multiplicity $\geq 3$ and that the space $\mathcal{M}_{6}^{b}$ of stable binary sextics exists (see [7]). In this section, we present the construction of Stephanos (see [1] -in a slightly different set up) associating to every Desargues configuration $D_{\pi}$ a binary sextic $J_{\pi}$ in a canonical way. In Section 5 we will see that $J_{\pi}$ is stable so that we get a holomorphic map $\Phi: M_{D} \longrightarrow \mathcal{M}_{6}^{b}$.

As in the last section we fix the coordinate system of $\mathbb{P}_{3}$ in such a way that $e_{1}=(1$ : $0: 0: 0), \ldots, e_{4}=(0: 0: 0: 1)$ and $e_{5}=(1: 1: 1: 1)$. Let $\pi$ be a nondegenerate plane in $\mathbb{P}_{3}$, meaning that the configuration $D_{\pi}$ is nondegenerate, with equation $\sum_{i=1}^{4} \alpha_{i} z_{i}=0$. For the coefficients $\alpha_{i}$ of $\pi$ this just means $\alpha_{i} \neq 0$ for $i=1, \ldots, 4$ and $\sum_{i=1}^{4} \alpha_{i} \neq 0$. In 1847, von Staudt proved in his fundamental book [10] that there is a unique (smooth) conic $s_{\pi}$ on $\pi$ such that the polar line of every point of the configuration $D_{\pi}$ is its axis. We call $s_{\pi}$ the von Staudt conic of $D_{\pi}$.

Lemma 3.1. The von Staudt conic $s_{\pi}$ of $D_{\pi}$ is given by the equation $\sum_{i=1}^{4} \alpha_{i} z_{i}^{2}=0$ in $\pi: \sum_{i=1}^{4} \alpha_{i} z_{i}=0$

PRoOF: According to a remark of Reye, which is easy to check (see [8],p. 135), there is a unique quadric $S_{5}$ in $\mathbb{P}_{3}$ such that the tetrahedron $e_{1}, e_{2}, e_{3}, e_{4}$ is a polar tetrahedron, i.e. the polar plane of $e_{i}$ with respect to $S_{5}$ is the opposite plane of the tetrahedron, and such that the polar plane of $e_{5}$ is the plane $\pi$. If, as usual, we denote by $S_{5}$ also the matrix of the quadric, the conditions mean $e_{i} S_{5} e_{j}=0$ for $1 \leq i, j \leq 4, i \neq j$ and $e_{5} S_{5}=\left(\alpha_{1}, \alpha_{2}, \alpha_{3}, \alpha_{4}\right)^{t}$. But this implies $S_{5}=\operatorname{diag}\left(\alpha_{1}, \alpha_{2}, \alpha_{3}, \alpha_{4}\right)$. Comparing the definitions, the von Staudt conic $s_{\pi}$ is just the restriction of $S_{5}$ to $\pi$, which gives the assertion.

Note that the proof of Lemma 3.1 yields actually a proof of von Staudt's Theorem. Obviously $S_{5}$ depends on the choice of $e_{5}$ as the cone vertex. One could in an analogous way define $S_{i}(1 \leq i \leq 4)$ and use it to prove Lemma 3.1.

As remarked in the last section, each point $e_{i}$ corresponds to a complete quadrangle of $D_{\pi}$, namely $p_{1 i}, \ldots, \check{p_{i i}}, \ldots, p_{5 i}$. Since these 4 points are in general position, there is 
a unique pencil of conics $\lambda q_{1}+\mu q_{2}$ passing through the 4 points. The pencil $\lambda q_{1}+\mu q_{2}$ restricts to a pencil of effective divisors of degree 4 on the von Staudt conic $s_{\pi}$. The Jacobian of this pencil is an effective divisor $j_{\pi}$ of degree 6 on $s_{\pi}$. It is defined as follows: choose an isomorphism $\varphi: s_{\pi} \longrightarrow \mathbb{P}_{1}$ and coordinates $\left(x_{1}, x_{2}\right)$ of $\mathbb{P}_{1}$. There are binary quartics $f_{1}\left(x_{1}, x_{2}\right)$ and $f_{2}\left(x_{1}, x_{2}\right)$ with zero divisor $\varphi\left(q_{1} \cap s_{\pi}\right)$ and $\varphi\left(q_{2} \cap s_{\pi}\right)$. The Jacobian of $f_{1}$ and $f_{2}$

$$
J_{\pi}\left(x_{1}, x_{2}\right)=\operatorname{det}\left(\frac{\partial f_{i}}{\partial x_{j}}\right)
$$

is a binary sextic with zero divisor $\varphi\left(j_{\pi}\right)$. Note the binary sextic is determined by the pencil $\lambda q_{1}+\mu q_{2}$ up to a nonzero constant and an automorphism of $\mathbb{P}_{1}$. We call $J_{\pi}$ the Jacobian sextic of the pencil, for the Jacobian divisor $j_{\pi}$ on $s_{\pi}$.

Theorem 3.2. The Jacobian divisor $j_{\pi}$ on the von Staudt conic $s_{\pi}$ is uniquely determined by the Desargues configuration $D_{\pi}$. It is cut out on $s_{\pi}$ by the cubic $\sum_{i=1}^{4} \alpha_{i} z_{i}^{3}=0$.

Proof: Choose first $i=5$. The pencil of conics passing through the 4 points $p_{15}, p_{25}, p_{35}, p_{45}$ is cut out on $\pi$ by the pencil of cones in $\mathbb{P}_{3}$ passing through the 4 lines $\overline{e_{1} e_{5}}, \overline{e_{2} e_{5}}, \overline{e_{3} e_{5}}, \overline{e_{4} e_{5}}$. The conditions for a quadric $Q \subset \mathbb{P}_{3}$ to be in this pencil are: $Q$ is singular in $e_{5}$, i.e. $Q e_{5}=0$ and $e_{i} \in Q$ for $i=1,2,3,4$, i.e. $e_{i} Q e_{i}=0$. This yields

$$
Q=\left(\begin{array}{cccc}
0 & a & b & -(a+b) \\
a & 0 & -(a+b) & b \\
b & -(a+b) & 0 & a \\
-(a+b) & b & a & 0
\end{array}\right)
$$

Hence the pencil is generated by the cones

$$
Q_{1}=\left(\begin{array}{cccc}
0 & 1 & 0 & -1 \\
1 & 0 & -1 & 0 \\
0 & -1 & 0 & 1 \\
-1 & 0 & 1 & 0
\end{array}\right) \text { and } Q_{2}=\left(\begin{array}{cccc}
0 & 0 & 1 & -1 \\
0 & 0 & -1 & 1 \\
1 & -1 & 0 & 0 \\
-1 & 1 & 0 & 0
\end{array}\right)
$$

It is easy to see that the Jacobi divisor of the pencil $\lambda q_{1}+\mu q_{2}$ on the curve $s_{\pi}=$ $\left\{\left(z_{1}: \ldots: z_{4}\right) \in \mathbb{P}_{3} \mid \sum_{i=1}^{4} \alpha_{i} z_{i}=\sum_{i=1}^{4} \alpha_{i} z_{i}^{2}=0\right\}$ is the intersection of the curve $s_{\pi}$ with the hypersurface of points in $\mathbb{P}_{3}$ where the vectors of partial derivatives $d\left(\sum \alpha_{i} z_{i}\right)$, $d\left(\sum \alpha_{i} z_{i}^{2}\right), d\left(Q_{1}\right), d\left(Q_{2}\right)$ are linearly dependent. Here we consider $Q_{i}$ as the quadratic form determined by the matrix $Q_{i}$. This hypersurface is given by the equation:

$$
J:=\operatorname{det}\left(\begin{array}{cccc}
\alpha_{1} & \alpha_{1} z_{1} & z_{2}-z_{4} & z_{3}-z_{4} \\
\alpha_{2} & \alpha_{2} z_{2} & z_{1}-z_{3} & z_{4}-z_{3} \\
\alpha_{3} & \alpha_{3} z_{3} & z_{4}-z_{2} & z_{1}-z_{2} \\
\alpha_{4} & \alpha_{4} z_{4} & z_{3}-z_{1} & z_{2}-z_{1}
\end{array}\right)=0
$$

We claim that $J$ is congruent to $\sum_{i=1}^{4} \alpha_{i} z_{i}^{3}$ modulo the ideal $\left(\sum_{i} \alpha_{i} z_{i}, \sum_{i} \alpha_{i} z_{i}^{2}\right)$. But

$$
J=\operatorname{det}\left(\begin{array}{cccc}
\alpha_{1} & \alpha_{1} z_{1} & z_{2}-z_{4} & z_{3}-z_{4} \\
\alpha_{2} & \alpha_{2} z_{2} & z_{1}-z_{3} & z_{4}-z_{3} \\
\alpha_{3} & \alpha_{3} z_{3} & z_{4}-z_{2} & z_{1}-z_{2} \\
\sum \alpha_{i} & \sum \alpha_{i} z_{i} & 0 & 0
\end{array}\right)
$$




$$
\begin{aligned}
\equiv & -\sum_{i} \alpha_{i} \operatorname{det}\left(\begin{array}{ccc}
\alpha_{1} z_{1} & z_{2}-z_{4} & z_{3}-z_{4} \\
\alpha_{2} z_{2} & z_{1}-z_{3} & z_{4}-z_{3} \\
\alpha_{3} z_{3} & z_{4}-z_{2} & z_{1}-z_{2}
\end{array}\right) \quad\left(\bmod \sum_{i} \alpha_{i} z_{i}\right) \\
= & -\sum_{i} \alpha_{i}\left[2\left(\alpha_{1} z_{1}^{3}+\alpha_{2} z_{2}^{3}+\alpha_{3} z_{3}^{3}\right)-\left(\alpha_{1} z_{1}^{2}-\alpha_{2} z_{2}^{2}-\alpha_{3} z_{3}^{2}\right) z_{4}-z_{4}\left(\alpha_{1} z_{1}^{2}+\alpha_{2} z_{2}^{2}+\alpha_{3} z_{3}^{2}\right)\right. \\
& \left.+\left(z_{1}+z_{2}+z_{3}\right)\left(z_{4}\left(\alpha_{1} z_{1}+\alpha_{2} z_{2}+\alpha_{3} z_{3}\right)-\left(\alpha_{1} z_{1}^{2}+\alpha_{2} z_{2}^{2}+\alpha_{3} z_{3}^{2}\right)\right)\right] \\
\equiv & -2 \sum_{i=1}^{4} \alpha_{i} \sum_{j=1}^{4} \alpha_{j} z_{j}^{3} \quad\left(\bmod \left(\sum_{i} \alpha_{i} z_{i}, \sum_{i} \alpha_{i} z_{i}^{2}\right)\right)
\end{aligned}
$$

It remains to show that if we start with another point $e_{i}$ we obtain the same Jacobi divisor $j_{\pi}$ on $s_{\pi}$. Without loss of generality we may start with $e_{1}$, since choosing another $e_{i}(2 \leq i \leq 4)$ amounts only to a permutation of the coordinates.

The pencil of conics in $\pi$ passing through the points $p_{12}, p_{13}, p_{14}, p_{15}$ is cut out on $\pi$ by the pencil of cones in $\mathbb{P}_{3}$ passing through the lines $\overline{e_{1} e_{2}}, \overline{e_{1} e_{3}}, \overline{e_{1} e_{4}}, \overline{e_{1} e_{5}}$. This pencil is generated by the cones:

$$
Q_{1}^{\prime}=\left(\begin{array}{cccc}
0 & 0 & 0 & 0 \\
0 & 0 & 1 & 0 \\
0 & 1 & 0 & -1 \\
0 & 0 & -1 & 0
\end{array}\right) \text { and } Q_{2}^{\prime}=\left(\begin{array}{cccc}
0 & 0 & 0 & 0 \\
0 & 0 & 0 & 1 \\
0 & 0 & 0 & -1 \\
0 & 1 & -1 & 0
\end{array}\right)
$$

A very similar computation as the one above shows that modulo the ideal $\left(\sum \alpha_{i} z_{i}, \sum \alpha_{i} z_{i}^{2}\right)$ the hypersurface of points in $\mathbb{P}_{3}$ where the vectors of partial derivatives $d\left(\sum \alpha_{i} z_{i}\right), d\left(\sum \alpha_{i} z_{i}^{2}\right)$, $d\left(Q_{1}^{\prime}\right), d\left(Q_{2}^{\prime}\right)$ are linearly dependent is given by $\sum_{i=1}^{4} \alpha_{i} z_{i}^{3}$, which completes the proof of the theorem.

If $\pi^{\prime}$ is another nondegenerate plane in $\mathbb{P}_{3}$ such that the Desargues configurations $D_{\pi}$ and $D_{\pi^{\prime}}$ are isomorphic, it follows immediately from Lemma 2.1 and Theorem 3.2 that the isomorphism $\alpha: \pi \longrightarrow \pi^{\prime}$ with $\alpha\left(D_{\pi}\right)=D_{\pi^{\prime}}$ maps the Jacobi divisor $j_{\pi}$ on $s_{\pi}$ onto the Jacobi divisor $j_{\pi^{\prime}}$ on $s_{\pi^{\prime}}$.

In the sequel we will always interpret $j_{\pi}$ as the binary sextic $J_{\pi}$ (see above and recall that $J_{\pi}$ is determined up to a nonzero constant and up to an automorphism of $\mathbb{P}_{1}$ ). In Theorem 5.1, we will show that $J_{\pi}$ is always stable. Hence we obtain a canonical map

$$
\Phi: M_{D} \longrightarrow \mathcal{M}_{6}^{b}
$$

of the moduli space of Desargues configurations into the moduli space of stable binary sextics. It is clear that $\Phi$ is holomorphic, since Theorem 3.2 implies that it is given by polynomials.

Remark 3.3. The proof of Theorem 3.2 suggests another definition of the Jacobi divisor $j_{\pi}$ : the von Staudt conic $s_{\pi}$ is not contained in the pencil of conics $\lambda q_{1}+\mu q_{2}$ passing through the points $p_{15}, p_{25}, p_{35}, p_{45}$. Hence $\lambda q_{1}+\mu q_{2}+\nu s_{\pi}$ is a net of conics in the plane $\pi$. Its discriminant locus given by

$$
\operatorname{det}\left(q_{1}, q_{2}, s_{\pi}\right)=0
$$

is a cubic which intersects the conic $s_{\pi}$ in the Jacobian divisor $j_{\pi}$. For the proof note only that the net is general and thus its discriminant is equivalent to its Jacobian locus given by $\operatorname{det}\left(d q_{1}, d q_{2}, d s_{\pi}\right)=0$. 
Remark 3.4. Given a nondegenerate plane $\pi$, we associated to every point $e_{i},(1 \leq i \leq 5)$ a pencil of quartic divisors $\lambda f_{1}^{i}+\mu f_{2}^{i}$ on the von Staudt conic $s_{\pi}$. It is easy to check that in general these 5 pencils are different from each other.

\section{$4 \quad$ Injectivity of $\Phi: M_{D} \longrightarrow \mathcal{M}_{6}^{b}$}

The main object of this section is to prove the following theorem.

Theorem 4.1. The map $\Phi: M_{D} \longrightarrow \mathcal{M}_{6}^{b}$ is an injective birrational morphism.

For the proof we need some preliminaries. Let $A$ and $B$ denote two quadrics in $\mathbb{P}_{n}$ (for us $n=2$ or 3 ) given by the equations $x^{t} A x=0$ and $x^{t} B x=0$. Recall that $A$ is called apolar to $B$ if

$$
\operatorname{tr}(A \cdot \operatorname{Adj}(B))=0
$$

Here $\operatorname{adj}(B)$ denotes the adjoint matrix of $B$, i.e. the matrix of the dual quadric $\hat{B}$ of $B$. Note that this definition is not symmetric (some authors say that $A$ is apolar to $\hat{B}$ ). Geometrically this means the following (see [9]): a conic $A$ is apolar to $B$ in $\mathbb{P}_{2}$ if and only if there is a triangle inscribed in $A$ and self-polar with respect to $B$. A quadric $A$ is apolar to $B$ in $\mathbb{P}_{3}$ if and only if there is a tetrahedron inscribed in $A$ and self-polar with respect to $B$.

Proposition 4.2. Let $D_{\pi}$ be a Desargues configuration. Every conic in $\pi$ passing through the 4 points of a complete quadrangle of $D_{\pi}$ is apolar to the von Staudt conic $s_{\pi}$.

PRoOF: Since apolarity is a linear condition and by the special choice of the coordinates it suffices to show that the quadric cones $Q_{1}, Q_{2}, Q_{1}^{\prime} Q_{2}^{\prime}$ (for the notation see proof of Theorem 3.2) are apolar to the the quadric diag $\left(\alpha_{1}, \alpha_{2}, \alpha_{3}, \alpha_{4}\right)$ in $\mathbb{P}_{3}$, whose restriction to $\pi$ is $s_{\pi}$, which is an immediate computation.

In order to show that a conic $q$ in the plane $\pi$ passing through the points of a complete quadrangle of $D_{\pi}$ is the unique conic apolar to $s_{\pi}$ and passing through $q \cap s_{\pi}$ (with multiplicities) we change the coordinates. We choose the coordinates $\left(x_{0}: x_{1}: x_{2}\right)$ of $\pi=\mathbb{P}_{2}$ in such a way that the von Staudt conic $s_{\pi}$ is given by the equation

$$
x_{1}^{2}-4 x_{0} x_{2}=0
$$

So the matrix of the dual conic is:

$$
\widehat{s_{\pi}}=\left(\begin{array}{ccc}
0 & 0 & 2 \\
0 & -4 & 0 \\
2 & 0 & 0
\end{array}\right)
$$

Let $\left(t_{0}: t_{1}\right)$ denote homogeneous coordinates of $\mathbb{P}_{1}$. Then

$$
\left(t_{0}: t_{1}\right) \longrightarrow\left(x_{0}: x_{1}: x_{2}\right)=\left(t_{0}^{2}: 2 t_{0} t_{1}: t_{1}^{2}\right)
$$


is a parametrization $\mathbb{P}_{1} \stackrel{\sim}{\longrightarrow} s_{\pi}$ of $s_{\pi}$. Using this, any effective divisor of degree 4 on $s_{\pi}$ can be interpreted as a binary quartic $f\left(t_{0}, t_{1}\right)$. Hence

$$
f\left(t_{0}, t_{1}\right)=a_{0} t_{0}^{4}+4 a_{1} t_{0}^{3} t_{1}+6 a_{2} t_{0}^{2} t_{1}^{2}+4 a_{3} t_{0} t_{1}^{3}+a_{4} t_{1}^{4}
$$

with $\left(a_{0}: a_{1}: a_{2}: a_{3}: a_{4}\right) \in \mathbb{P}_{5}$ representing an arbitrary divisor of degree 4 on $s_{\pi}$. Given an effective divisor $\delta$, of degree 4 on $s_{\pi}$, i.e. a binary quartic $f$, consider the conic

$$
q=a_{0} x_{0}^{2}+a_{2} x_{1}^{2}+a_{4} x_{2}^{2}+2 a_{1} x_{0} x_{1}+2 a_{2} x_{0} x_{2}+2 a_{3} x_{1} x_{2} .
$$

Lemma 4.3. The conic $q$ is the unique conic passing through the divisor $\delta$ and apolar to the von Staudt conic $s_{\pi}$.

Proof: Note first that $q$ passes through $\delta$ by the choice of the parametrization. It is apolar to $s_{\pi}$, since

$$
\operatorname{tr}\left(\left(\begin{array}{lll}
a_{0} & a_{1} & a_{2} \\
a_{1} & a_{2} & a_{3} \\
a_{2} & a_{3} & a_{4}
\end{array}\right)\left(\begin{array}{ccc}
0 & 0 & 2 \\
0 & -4 & 0 \\
2 & 0 & 0
\end{array}\right)\right)=2 a_{2}-4 a_{2}+2 a_{2}=0 .
$$

On the other hand, apolarity is one linear condition. Hence the space of conics apolar to $s_{\pi}$ is isomorphic to $\mathbb{P}_{4}$. Now it is easy to see ( $[3]$, p.17) that any divisor of degree 4 on $s_{\pi}$ imposes independent conditions on conics. In other words, the space of conics passing through $\delta$ is isomorphic to $\mathbb{P}_{1}$. Hence there is exactly one conic passing through $\delta$ and apolar to $s_{\pi}$ if we only show that not every conic passing through $\delta$ is apolar to $s_{\pi}$.

For this we assume $\delta=p_{1}+p_{2}+p_{3}+p_{4}$ with $p_{i} \neq p_{j}$, for $i \neq j$, the degenerate cases being even easier to check. We may choose the coordinates in such a way that in $\mathbb{P}_{1}$ we have $p_{1}=(0: 1), p_{2}=(1: 0), p_{3}=(1: 1), p_{4}=(1: t)$ with $t \neq 0,1$. So in $\mathbb{P}_{2}: p_{1}=(0: 0: 1), p_{2}=(1: 0: 0), p_{3}=(1: 2: 1), p_{4}=\left(1: 2 t: t^{2}\right)$ then $2 t(t-1) x y+\left(1-t^{2}\right) y^{2}+(2 t-2) y z=0$ is a conic passing through $p_{i}$, for $i=1, \ldots, 4$ and not apolar to $s_{\pi}$, if $t \neq-1$.

If $t=-1$ then $2 x y+4 x z-y^{2}-2 y z$ satisfies these conditions.

We call $q$ the conic associated to the binary quartic (2). If we associate to every divisor of a pencil $\lambda \delta_{1}+\mu \delta_{2}$ of quartic divisors the unique conic of Lemma 4.3, we obtain a pencil of conics. Hence we obtain as an immediate consequence of Proposition 4.2 and Lemma 4.3:

Corollary 4.4. Let $D_{\pi}$ be a Desargues configuration. The pencil of conics $\lambda q_{1}+\mu q_{2}$ passing through the 4 points of a complete quadrangle of $D_{\pi}$ is the unique pencil of conics cutting out the pencil of quartic divisors $\left(\lambda q_{1}+\mu q_{2}\right) \cap s_{\pi}$ which is apolar to the von Staudt conic $s_{\pi}$.

Given a smooth conic $q_{0}$, we choose the coordinates in such a way that $q_{0}$ is given by equation (1). As outlined above, any pencil of binary quartics can be interpreted (up to isomorphism) as a pencil of quartic divisors on $q_{0}$. There is a unique pencil of conics associated to it according to Lemma 4.3. We call a pencil of binary quartics (respectively the corresponding pencil of quartic divisors) admissible if its associated pencil of conics is general, i.e. its base locus consists of 4 different points. The following proposition is the first step in the proof of Theorem 4.1. 
Proposition 4.5. Consider an admissible pencil of quartic divisors on a smooth conic $q_{0}$. There is a unique Desargues configuration $D$ such that $q_{0}$ is the von Staudt conic of $D$ and the pencil of quartics is cut out on $q_{0}$ by the pencil of conics passing through the points of a complete quadrangle of $D$.

Proof: Let $p_{1}, p_{2}, p_{3}, p_{4}$ denote the 4 base points of the pencil of conics associated to the given pencil of quartic divisors. Consider the complete quadrangle consisting of the 4 points $p_{1}, p_{2}, p_{3}, p_{4}$ and the 6 lines $\ell_{i j}=\overline{p_{i} p_{j}}$ for $1 \leq i<j \leq 4$. Let $p_{i j}$ be the poles of the lines $\ell_{i j}$ and $\ell_{i}$ the polars of the points $p_{i}$ with respect to the conic $q_{0}$. Then the 10 points $P_{i j}, p_{i}$ and the 10 lines $\ell_{i j}, \ell_{i}$ form a Desargues configuration according to a theorem proved by von Staudt in 1831 (see [2], p.62). Using Lemma 4.3 we have the assertion.

Before we go on, let us note the following characterization of admissible binary quartics, which we need later.

Proposition 4.6. The pencil of binary quartics $\lambda f_{1}+\mu f_{2}$ with $f_{i}=\sum_{j=1}^{4}\left(\begin{array}{c}4 \\ i\end{array}\right) a_{j}^{i} t_{0}^{4-j} t_{1}^{j}$ is admissible if and only if the discriminant of the binary cubic $\operatorname{det}\left(t_{0} q_{1}+t_{1} q_{2}\right)$ is nonzero, where $q_{i}=\left(\begin{array}{ccc}a_{0}^{i} & a_{1}^{i} & a_{2}^{i} \\ a_{1}^{i} & a_{2}^{i} & a_{3}^{i} \\ a_{2}^{i} & a_{3}^{i} & a_{4}^{i}\end{array}\right)$ is the matrix of the conic associated to f for $i=1,2$.

Proof: This is a consequence of the fact (see [12], Section 6.3,) that the base locus of a pencil of conics consists of 4 different points if and only if its discriminant does not vanish together with Lemma 4.3.

In order to complete the proof of Theorem 4.1, we need to compute the degree of the Jacobian map. Note that the binary quartic (2) determines a point in $\mathbb{P}_{4}$ namely $\left(a_{0}: \ldots: a_{4}\right)$. If we consider $\mathbb{P}_{4}$ as the space of quartics, the space of pencils of binary quartics is $\operatorname{Gr}(1,4)$ the Grassmannian of lines in $\mathbb{P}_{4}$. Considering in the same way the space of binary sextics $\sum_{i=0}^{6} a_{i} t_{0}^{6-i} t_{1}^{i}$ as $\mathbb{P}_{6}$, the map associating to every pencil of quartics its Jacobian defines a morphism

$$
J a c: G r(1,4) \longrightarrow \mathbb{P}_{6}
$$

Note that $\operatorname{Gr}(1,4)$ is also of dimension 6 . We need the following lemma:

Lemma 4.7. The Jacobian map Jac: $\operatorname{Gr}(1,4) \longrightarrow \mathbb{P}_{6}$ is a ramified covering of degree 5 .

PRoOF: Let $\lambda f_{1}+\mu f_{2}$ be a pencil of quartics with $f_{1}=\sum_{i=1}^{4}\left(\begin{array}{l}4 \\ i\end{array}\right) a_{i} t_{0}^{4-i} t_{1}^{i}$ and $f_{2}=$ $\sum_{i=1}^{4}\left(\begin{array}{l}4 \\ i\end{array}\right) b_{i} t_{0}^{4-i} t_{1}^{i}$. Its Jacobian is given by:

$$
\begin{gathered}
\operatorname{Jac}\left(f_{1}, f_{2}\right)=\operatorname{det}\left(\frac{\partial f_{i}}{\partial t_{j}}\right)= \\
=16\left[p_{01} t_{0}^{6}+3 p_{02} t_{0}^{5} t_{1}+3\left(2 p_{12}+p_{03}\right) t_{0}^{4} t_{1}^{2}+\left(8 p_{13}+p_{04}\right) t_{0}^{3} t_{1}^{3}+3\left(2 p_{23}+p_{14}\right) t_{0}^{2} t_{1}^{4}+3 p_{24} t_{0} t_{1}^{5}+p_{34} t_{1}^{6}\right],
\end{gathered}
$$
where $p_{i j}:=a_{i} b_{j}-a_{j} b_{i}$ denotes the Plücker coordinates of the pencil. In particular up to a constant the Jacobian does not depend on the choice of $f_{1}$ and $f_{2}$ and defines a point $\operatorname{Jac}\left(\lambda f_{1}+\mu f_{2}\right)$ in $\mathbb{P}_{6}$. The explicit form of $J a c\left(f_{1}, f_{2}\right)$ implies that the map $J a c$ factorizes via the Plücker embedding $p$ 


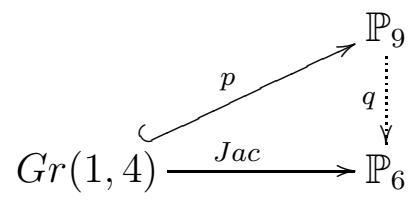

with a linear projection map $q$. In fact $J a c$ is given by

$$
\lambda f_{1}+\mu f_{2} \mapsto\left(p_{01}: 3 p_{02}: 3\left(2 p_{12}+p_{03}\right): 8 p_{13}+p_{04}: 3\left(2 p_{23}+p_{14}\right): 3 p_{24}: p_{34}\right) \in \mathbb{P}_{6}
$$

It is the linear projection of the $\mathbb{P}_{9}$ with coordinates $\left(p_{01}: \ldots: p_{34}\right)$ with center the plane $P$ with equations $p_{01}=p_{02}=2 p_{12}+p_{03}=8 p_{13}+p_{04}=2 p_{23}+p_{14}=p_{24}=p_{34}=0$. On the other hand, the Plücker variety $p(G r(1,4))$ in $\mathbb{P}_{9}$ is given by the Plücker relations among which, there are

$$
\begin{aligned}
& p_{12} p_{34}-p_{13} p_{24}+p_{14} p_{23}=0 \\
& p_{01} p_{34}-p_{03} p_{14}+p_{04} p_{13}=0 \\
& p_{01} p_{23}-p_{02} p_{13}+p_{03} p_{12}=0
\end{aligned}
$$

Now if $\left(p_{01}: \ldots: p_{34}\right) \in P \cap p(G r(1,4))$, this implies $p_{14} p_{23}=p_{04} p_{13}=p_{03} p_{12}=0$, so all $p_{i j}=0$. Hence the center of projection $P$ does no intersect the Plücker variety. This implies that the degree of $J a c$ equals the degree of the Plücker variety $p(\operatorname{Gr}(1,4))$ in $\mathbb{P}_{9}$. It is well known that this degree is 5 (see [6],p. 247). Since Jac cannot contract a positive dimensional subvariety of $\operatorname{Gr}(1,4)$ this completes the proof of the lemma.

Using this we can finally prove Theorem 4.1.

Proof of Theorem 4.1: Since admissibility of a pencil of quartics is an open condition and the Jacobian map is finite, we conclude from Proposition 4.5 that $\Phi$ is dominant and there is an open dense set $U \subset \mathcal{M}_{6}^{b}$ such that for every sextic $j \in U$ the preimage $J a c^{-1}(j)$ consists of exactly 5 pencils of quartics. Shrinking $U$ if necessary we may assume according to Remark 3.4 that every Desargues configuration $D \in \Phi^{-1}(U)$ admits 5 different pencils of quartic divisors on the von Staudt conic. Hence for every $j \in U$ the 5 pencils of quartics of any preimage $D \in \Phi^{-1}(j)$ must coincide. Thus by Proposition $4.5 \Phi^{-1}(j)$ consists only of one Desargues configuration, i.e. $\Phi: \Phi^{-1}(U) \longrightarrow U$ is bijective. This implies that $\Phi$ is of degree 1 . It remains to show the injectivity.

Since $\Phi$ is a finite birrational morphism of normal varieties, the injectivity can only fail at points $p$ of $M_{D}$ for which $\Phi(p)$ is a singular point of $\mathcal{M}_{6}^{b}$. But $\mathcal{M}_{6}^{b}$ admits only 3 singular points (see $\mathbb{1}$, Remark 5.4, p.5551), namely a point of mutiplicity 5 represented by the binary sextic $j_{5}=t_{0}^{6}-t_{0} t_{1}^{5}$, a point of multiplicity 2 represented by $j_{2}=t_{0}^{6}-t_{0}^{2} t_{1}^{4}$, a point of multiplicity 3 represented by $j_{3}=t_{0}^{5} t_{1}-t_{0}^{2} t_{1}^{4}$. The moduli space $M_{D}$ also admits a singular point of multiplicity 5: the Desargues configuration $D_{\pi_{5}}$ with $\pi_{5}$ given by $z_{1}-\mu z_{2}+\mu^{2} z_{3}-\mu^{3} z_{4}=0$ where $\mu$ is a root of the equation $x^{4}+x^{3}-x^{2}+x-1=0$, admits an automorphism of order 5 namely:

$$
\left(\begin{array}{cccc}
0 & 0 & 0 & 1 \\
-1 & 0 & 0 & 1 \\
0 & -1 & 0 & 1 \\
0 & 0 & -1 & 1
\end{array}\right)
$$


It permutes the points $e_{i}$ as follows: $e_{1} \mapsto e_{2} \mapsto e_{3} \mapsto e_{4} \mapsto e_{5} \mapsto e_{1}$. It is easy to check that $D_{\pi_{5}}$ is of multiplicity 5 in $M_{d}$ and that $\Phi^{-1}\left(j_{5}\right)=D_{\pi_{5}}$.

As in Example 5.3 below one checks that all pencils of quartics with Jacobian $j_{2}$ are not admissible. Hence $j_{2}$ is not in the image of $\Phi$. Finally as in Example 5.4 below one checks that only one pencil of quartics with Jacobian $j_{3}$ is admissible namely $\lambda f_{3}+\mu g_{3}$ with $f_{3}=2 t_{0}^{4}+6 t_{0}^{2} t_{1}^{2}+4 t_{0} t_{1}^{3}$ and $g_{3}=\frac{1}{16} t_{0}^{2} t_{1}^{2}$. Hence $\Phi$ is also injective at the corresponding point at $M_{D}$. This completes the proof of the theorem.

\section{The image of the map $\Phi$}

Using the set up of the last section we are now in a position to prove the following theorem, which was anounced (but not applied) already in Section 3.

Theorem 5.1. For any Desargues configuration $D$ the associated binary sextic $J=\Phi(D)$ is stable.

Proof: Let $J\left(t_{0}, t_{1}\right)$ be a nonstable binary sextic, i.e admitting a root of multiplicity $\geq 3$. We choose coordinates in such a way that this root is $(0: 1)$. Hence $J$ is of the form

$$
J\left(t_{0}, t_{1}\right)=A_{0} t_{0}^{6}+A_{1} t_{0}^{5} t_{1}+A_{2} t_{0}^{4} t_{1}^{2}+A_{3} t_{0}^{3} t_{1}^{3} .
$$

Suppose $\lambda f+\mu g$ with $f=\sum_{i=1}^{4}\left(\begin{array}{l}4 \\ i\end{array}\right) a_{i} t_{0}^{4-i} t_{1}^{i}$ and $g=\sum_{i=1}^{4}\left(\begin{array}{l}4 \\ i\end{array}\right) b_{i} t_{0}^{4-i} t_{1}^{i}$ is a pencil of binary quartics whose Jacobian is $J$ (up to a nonzero constant). According to the results of Section 4 , it suffices to show that $\lambda f+\mu g$ is not admissible which according to Proposition 4.6 means that $\operatorname{det}\left(t_{0} q_{1}+t_{1} q_{2}\right)$ admits a double root where $q_{1}$ and $q_{2}$ denote the conics associated to $f$ and $g$. By the equation for $J$ given in the proof of Lemma 4.7 the coefficients of $f$ and $g$ satisfy the following system of equations

$$
\begin{aligned}
2\left(a_{2} b_{3}-a_{3} b_{2}\right)+a_{1} b_{4}-a_{4} b_{1} & =0 \\
a_{2} b_{4}-a_{4} b_{2} & =0 \\
a_{3} b_{4}-a_{4} b_{3} & =0
\end{aligned}
$$

Assume first that $a_{4}$ or $b_{4} \neq 0$. Without loss of generality we may assume that $a_{4}=1$. Replacing $g$ by $g-b_{4} f$ we may assume $b_{4}=0$. But then the system (3) implies $b_{3}=b_{2}=$ $b_{1}=0$ and we obtain

$$
\operatorname{det}\left(t_{0} q_{1}+t_{1} q_{2}\right)=\left(\begin{array}{ccc}
t_{0} a_{0}+t_{1} b_{0} & t_{0} a_{1} & t_{0} a_{2} \\
t_{0} a_{1} & t_{0} a_{2} & t_{0} a_{3} \\
t_{0} a_{2} & t_{0} a_{3} & t_{0}
\end{array}\right)
$$

which has a double root $(0: 1)$. Hence $a_{4}=b_{4}=0$. Then (3) just says

$$
a_{2} b_{3}-a_{3} b_{2}=0
$$

If $a_{3}=b_{3}=0$,

$$
\operatorname{det}\left(t_{0} q_{1}+t_{1} q_{2}\right)=\left(\begin{array}{ccc}
t_{0} a_{0}+t_{1} b_{0} & t_{0} a_{1}+t_{1} b_{1} & t_{0} a_{2}+t_{1} b_{2} \\
t_{0} a_{1}+t_{1} b_{1} & t_{0} a_{2}+t_{1} b_{2} & 0 \\
t_{0} a_{2}+t_{1} b_{2} & 0 & 0
\end{array}\right)
$$


which has a triple root. Finally, again without loss of generality, we may assume that $a_{3}=1, b_{3}=0$. Then (41) implies $b_{2}=0$ and

$$
\operatorname{det}\left(t_{0} q_{1}+t_{1} q_{2}\right)=\left(\begin{array}{ccc}
t_{0} a_{0}+t_{1} b_{0} & t_{0} a_{1}+t_{1} b_{1} & t_{0} a_{2} \\
t_{0} a_{1}+t_{1} b_{1} & t_{0} a_{2} & t_{0} \\
t_{0} a_{2} & t_{0} & 0
\end{array}\right)
$$

which again has a double root $(0: 1)$. This completes the proof of the theorem.

Theorems 4.1 and 5.1 lead to the question whether the map $\Phi: M_{D} \longrightarrow \mathcal{M}_{6}^{b}$ is surjective. In order to analize this question, recall that we represented a quartic divisor on the conic $s: x_{1}^{2}-4 x_{0} x_{2}$ by the binary quartic:

$$
f\left(t_{0}, t_{1}\right)=a_{0} t_{0}^{4}+4 a_{1} t_{0}^{3} t_{1}+6 a_{2} t_{0}^{2} t_{1}^{2}+4 a_{3} t_{0} t_{1}^{3}+a_{4} t_{1}^{4}
$$

and the conic associated to it was given by the matrix $\left(\begin{array}{ccc}a_{0} & a_{1} & a_{2} \\ a_{1} & a_{2} & a_{3} \\ a_{2} & a_{3} & a_{4}\end{array}\right)$. We are identifying in this way the projective space $\mathbb{P}_{4}=\mathbb{P}_{4}\left(a_{0}: \ldots: a_{4}\right)$ of binary quartics with the $\mathbb{P}_{4}$ of conics apolar to the conic $s$. Similarly we consider $\operatorname{Gr}(1,4)$ as a space of pencils of conics. Hence we get a map

$$
J a c: G r(1,4) \longrightarrow \mathbb{P}_{6}
$$

associating to every pencil of conics apolar to $s$ the Jacobian of its associated pencil of binary quartics. According to the definition of the map $\Phi$ a binary sextic $j$ is in the image of $\Phi$ if and only if the pre-image $J_{a c}^{-1}(j)$ (which consists of 5 pencils counted with multiplicities) consists only of special pencils of conics, i.e. pencils of conics $\lambda q_{1}+\mu q_{2}$ such that $\operatorname{det}\left(\lambda q_{1}+\mu q_{2}\right)$ admits a multiple root $(\lambda: \mu)$. Let $\mathcal{D} \subset \operatorname{Gr}(1,4)$ denote the hypersurface of $\operatorname{Gr}(1,4)$ given by the equation

$$
\operatorname{discrim}\left(\operatorname{det}\left(\lambda q_{1}+\mu q_{2}\right)\right)=0
$$

$J a c$ being a finite morphism, the image $\operatorname{Jac}(\mathcal{D})$ is a hypersurface in $\mathbb{P}_{6}$. If $U$ denotes the open set of $\mathbb{P}_{6}$ parametrizing stable sextics and $\rho: U \longrightarrow \mathcal{M}_{6}^{b}$ the natural projection, it is easy to see that also

$$
\mathcal{H}:=p(\operatorname{Jac}(\mathcal{D}) \cap U)
$$

is a hypersurface in $\mathcal{M}_{6}^{b}$. We obtain

Proposition 5.2. The image $\Im m(\Phi)$ of $\Phi$ satisfies

$$
\mathcal{M}_{6}^{b} \backslash \mathcal{H} \subset \Im m(\Phi) \subset \mathcal{M}_{6}^{b}
$$

The following two examples show that both inclusions are strict, i.e. $\Im m(\Phi) \neq \mathcal{M}_{6}^{b}$ and $\mathcal{M}_{6}^{b} \backslash \mathcal{H} \neq \Im m(\Phi)$.

Example 5.3. Consider the binary sextic

$$
j_{0}=-16 t_{1}^{6}-48 t_{0}^{4} t_{1}^{2}+128 t_{0}^{3} t_{1}^{3}-48 t_{0}^{2} t_{1}^{4}-16 t_{1}^{6}
$$

$J_{a c^{-1}}\left(j_{0}\right)$ contains the following 3 pencils of conics $p_{i}=\lambda q_{1}^{i}+\mu q_{2}^{i},(i=1,2,3)$ with 


$$
\begin{aligned}
q_{1}^{1} & =\left(\begin{array}{ccc}
-1 & 0 & 0 \\
0 & 0 & 0 \\
0 & 0 & 1
\end{array}\right) \quad q_{2}^{1}=\left(\begin{array}{ccc}
-8 & 1 & 0 \\
1 & 0 & 1 \\
0 & 1 & 0
\end{array}\right) \\
q_{1}^{2} & =\left(\begin{array}{ccc}
1 & 1 & -1 \\
1 & -1 & 0 \\
-1 & 0 & 1
\end{array}\right) \quad q_{2}^{2}=\left(\begin{array}{lll}
0 & 1 & 0 \\
1 & 0 & 1 \\
0 & 1 & 0
\end{array}\right) \\
q_{1}^{3} & =\left(\begin{array}{ccc}
-1 & 1 & 0 \\
1 & 0 & 0 \\
0 & 0 & 1
\end{array}\right) \quad q_{2}^{3}=\left(\begin{array}{lll}
0 & 1 & 0 \\
1 & 0 & 1 \\
0 & 1 & 0
\end{array}\right)
\end{aligned}
$$

Since the Jacobian matrix of the map Jac: $\operatorname{Gr}(1,4) \longrightarrow \mathbb{P}_{6}$ at the points $p_{1}, p_{2}, p_{3}$ of $G r(1,4)$ is of rank 5,6,5 the pencils $p_{1}$ and $p_{3}$ are in the ramification locus of $J a c$. Hence $\operatorname{Jac}^{-1}\left(j_{0}\right)=\left\{p_{1}, p_{2}, p_{3}\right\}$. Now it is easy to check that $\operatorname{discr}\left(\operatorname{det}\left(\lambda q_{1}^{i}+\mu q_{2}^{i}\right)\right)=0$ for $i=1,2,3$. hence there is no Desargues configuration $D_{\pi}$ with $\Phi\left(D_{\pi}\right)=j_{0}$.

Example 5.4. Consider the binary sextic

$$
j_{1}=48 t_{0}^{5} t_{1}+48 t_{0}^{2} t_{1}^{4}
$$

$J_{a c}^{-1}\left(j_{1}\right)$ contains the 2 pencils of conics $p_{i}=\lambda q_{1}^{i}+\mu q_{2}^{i},(i=1,2)$ with

$$
\begin{gathered}
q_{1}^{1}=\left(\begin{array}{ccc}
2 \sqrt[3]{-2} & 1 & \frac{1}{8} \sqrt[3]{4} \\
1 & \frac{1}{8} \sqrt[3]{4} & \frac{1}{4} \sqrt[3]{-2} \\
\frac{1}{8} \sqrt[3]{4} & \frac{1}{4} \sqrt[3]{-2} & 1
\end{array}\right) \quad q_{2}^{1}=\left(\begin{array}{ccc}
2 \sqrt[3]{-2} & -1 & 0 \\
-1 & 0 & 0 \\
0 & 0 & 0
\end{array}\right) \\
q_{1}^{2}=\left(\begin{array}{ccc}
1 & 0 & 1 \\
0 & 1 & -\frac{1}{2} \\
1 & -\frac{1}{2} & 0
\end{array}\right) \quad q_{2}^{2}=\left(\begin{array}{lll}
0 & 0 & 1 \\
0 & 1 & 0 \\
1 & 0 & 0
\end{array}\right)
\end{gathered}
$$

One easily checks $\operatorname{discr}\left(\operatorname{det}\left(\lambda q_{1}^{1}+\mu q_{2}^{1}\right)\right)=0$ and $\operatorname{discr}\left(\operatorname{det}\left(\lambda q_{1}^{2}+\mu q_{2}^{2}\right)\right) \neq 0$. So $\lambda q_{1}^{2}+\mu q_{2}^{2}$ comes from a Desargues configuration, whereas $\lambda q_{1}^{1}+\mu q_{2}^{1}$ does not. This implies $j_{1} \in$ $\Im m(\Phi)$, but $j_{1} \in \mathcal{H}$.

\section{Special Desargues configurations}

Consider the 10 points in $\mathbb{P}_{3}$

$$
\begin{array}{ll}
S_{12}:=\overline{e_{1} e_{2}} \cap \overline{e_{3} e_{4} e_{5}}=(1: 1: 0: 0) & S_{34}:=\overline{e_{3} e_{4}} \cap \overline{e_{1} e_{2} e_{5}}=(0: 0: 1: 1) \\
S_{13}:=\overline{e_{1} e_{3}} \cap \overline{e_{2} e_{4} e_{5}}=(1: 0: 1: 0) & S_{15}:=\overline{e_{1} e_{5}} \cap \overline{e_{2} e_{3} e_{4}}=(0: 1: 1: 1) \\
S_{14}:=\overline{e_{1} e_{4}} \cap \overline{e_{2} e_{3} e_{5}}=(1: 0: 0: 1) & S_{25}:=\overline{e_{2} e_{5}} \cap \overline{e_{1} e_{3} e_{4}}=(1: 0: 1: 1)
\end{array}
$$




$$
\begin{array}{ll}
S_{23}:=\overline{e_{2} e_{3}} \cap \overline{e_{1} e_{4} e_{5}}=(0: 1: 1: 0) & S_{35}:=\overline{e_{3} e_{5}} \cap \overline{e_{1} e_{2} e_{4}}=(1: 1: 0: 1) \\
S_{24}:=\overline{e_{2} e_{4}} \cap \overline{e_{1} e_{3} e_{5}}=(0: 1: 0: 1) & S_{34}:=\overline{e_{3} e_{4}} \cap \overline{e_{1} e_{2} e_{5}}=(1: 1: 1: 0)
\end{array}
$$

Recall that a Desargues configuration $D_{\pi}$ is special if and only if the plane $\pi$ contains one of the points $S_{i j}$ (and no point $e_{i}$ ). The following theorem gives a characterization of special Desargues configurations in terms of their associated binary sextics.

Theorem 6.1. For a Desargues configuration $D_{\pi}$ the following statements are equivalent: (1) $D_{\pi}$ is a special Desargues configuration.

(2) The binary sextic $\Phi\left(D_{\pi}\right)$ admits a double point.

ProOF: Suppose $\pi$ given by $f(z)=\sum_{i=1}^{4} \alpha_{i} z_{i}=0$ is a nondegenerate plane in $\mathbb{P}_{3}$, such that $\Phi\left(D_{\pi}\right)$ admits a double point. According to Theorem $3.2 \Phi\left(D_{\pi}\right)$ is the sextic divisor on the von Staudt conic defined by the complete intersection $I(f, g, h)$ with $g(z)=\sum_{i=1}^{4} \alpha_{i} z_{i}^{2}=0$ and $h(z)=\sum_{i=1}^{4} \alpha_{i} z_{i}^{3}=0$. A point on this complete intersection scheme is a double point if and only if the rank of the Jacobian matrix $J(f, g, h)$ is $\leq 2$ at this point. But

$$
J(f, g, h)=\left(\begin{array}{c}
d f \\
d g \\
d h
\end{array}\right)=\left(\begin{array}{cccc}
\alpha_{1} & \alpha_{2} & \alpha_{3} & \alpha_{4} \\
2 \alpha_{1} z_{1} & 2 \alpha_{2} z_{2} & 2 \alpha_{3} z_{3} & 2 \alpha_{4} z_{4} \\
3 \alpha_{1} z_{1}^{2} & 3 \alpha_{2} z_{2}^{2} & 3 \alpha_{3} z_{3}^{2} & 3 \alpha_{4} z_{4}^{2}
\end{array}\right)
$$

So $r k(J(f, g, h))=r k\left(\begin{array}{cccc}1 & 1 & 1 & 1 \\ z_{1} & z_{2} & z_{3} & z_{4} \\ z_{1}^{2} & z_{2}^{2} & z_{3}^{2} & z_{4}^{2}\end{array}\right) \leq 2$ if and only if

$$
\operatorname{det}\left\{\left(\begin{array}{cccc}
1 & 1 & 1 & 1 \\
z_{1} & z_{2} & z_{3} & z_{4} \\
z_{1}^{2} & z_{2}^{2} & z_{3}^{2} & z_{4}^{2}
\end{array}\right) \text { with ith column omitted }\right\}=0
$$

for $i=1, \ldots, 4$. But these are Vandermonde determinants. Hence $\left(z_{1}: \ldots: z_{4}\right) \in \Phi\left(D_{\pi}\right)$ is a double point if and only if it satisfies the following system of equations

$$
\left\{\begin{array}{l}
\left(z_{1}-z_{2}\right)\left(z_{1}-z_{3}\right)\left(z_{2}-z_{3}\right)=0 \\
\left(z_{1}-z_{2}\right)\left(z_{1}-z_{4}\right)\left(z_{2}-z_{4}\right)=0 \\
\left(z_{1}-z_{3}\right)\left(z_{1}-z_{4}\right)\left(z_{3}-z_{4}\right)=0 \\
\left(z_{2}-z_{3}\right)\left(z_{2}-z_{4}\right)\left(z_{3}-z_{4}\right)=0
\end{array}\right.
$$

The solutions of the system above are exactly the points in $\mathbb{P}_{3}$ with 3 equal coordinates or two pairs of equal coordinates. So after normalizing one type of such points is represented by $(1: 1: 1: \gamma)$ and the other by $(1: 1: \gamma: \gamma)$ with $\gamma \in \mathbb{C}$. Such a point is contained in the complete intersection $I(f, g, h)$ if and only if $\gamma=1$ or $\gamma=0$. If $\gamma=1$ then $s_{\pi}$ contains the point $e_{5}$, so $\pi$ is degenerate. If $\gamma=0$ then the $\left(z_{1}: \ldots: z_{4}\right)$ is one of the 10 points $S_{i j}$ as above. We conclude that $\Phi\left(D_{\pi}\right)$ admits a double point if and only if $D_{\pi}$ is special.

Remark 6.2. In the same way one can show that a Desargues configuration $D_{\pi}$ admits 2 (respectively 3 ) lines containing 4 points if and only if $\Phi\left(D_{\pi}\right)$ admits 2 (respectively 3 ) double points. 


\section{Degenerate Desargues configuration}

In this section we study an extension of the map $\Phi: M_{D} \longrightarrow \mathcal{M}_{6}^{b}$ to a holomorphic map $\bar{\Phi}: U \longrightarrow \mathcal{M}_{6}^{b}$ where $U$ is an open set with $M_{D} \subset U \subset \bar{M}_{D}$, where the first inclusion is strict. First we have to define the von Staudt conic for degenerate Desargues configurations.

Recall that the von Staudt conic of a non degenerate Desargues configuration $D_{\pi}$ is defined by the fact that the map associating to every point $p_{i j}$ in $D_{\pi}$ the line $l_{k l m}$ with complementary indices is a polarity. This definition can be generalized verbatim to the case of generalized Desargues configurations. One only has to add that if 2 points $p_{i j}$ and $p_{k l}$ fall together, no line is associated to them.

To be more precise, let $D_{\pi}$ be of the first kind, i.e. $D_{\pi}$ contains exactly one of the points $e_{i}$. For the sake of notational simplicity we assume that $e_{5} \in \pi$ (see Figure 3 ). In this case the map associating to $p_{i j}(i, j \neq 5)$ the line $\ell_{k l 5}$ is just given by the involution $\imath$ on the pencil of lines through $e_{5}$ given by the fact that $D_{\pi}$ is a limit of Desargues configurations (see Section 1). This involution has 2 fixed lines and their union is the von Staudt conic $s_{\pi}$.

Let now $D_{\pi}$ be of the second kind. Without loss of generality, we assume the case of Figure 4 , i.e. $e_{4}$ and $e_{5} \in \pi$. There are only 3 points apart from $e_{4}$ and $e_{5}$ namely $p_{12}, p_{13}$ and $p_{23}$. To $p_{12}$ the line $\ell_{345}$ is associated, to $p_{13}$ the line $\ell_{245}$ and to $p_{23}$ the line $\ell_{145}$. But these 3 lines coincide with $\overline{e_{4} e_{5}}$. Hence the von Staudt conic $s_{\pi}$ in this case is the double line $\overline{e_{4} e_{5}}$.

Finally if $D_{\pi}$ is of the third kind, there are 3 points in the plane $\pi$ to which no line is associated. This implies that the von Staudt conic $s_{\pi}$ is the zero conic, i.e. the whole plane $\pi$. Note that if $D_{\pi}$ is of the $i^{t h}$ kind, the rank of the conic is $3-i$. Analogously to Lemma 3.1 we have:

Lemma 7.1. Suppose $D_{\pi}$ is a degenerate Desargues configuration. The von Staudt conic $s_{\pi}$ of $D_{\pi}$ is given by the equation $\sum_{i=1}^{4} \alpha_{i} z_{i}^{2}=0$ in the plane $\pi=\sum_{i=1}^{4} \alpha_{i} z_{i}=0$.

PRoOF: One can prove this either in the same way as Lemma 3.1 using an analogous version of Reyes remark (see proof of Lemma 3.1) or just check it by computation:

If $D_{\pi}$ is degenerate of the first kind with say $e_{4} \in \pi$, i.e. $\pi$ is given by $\alpha_{1} z_{1}+\alpha_{2} z_{2}+\alpha_{3} z_{3}=0$ with $\alpha_{1}, \alpha_{2}, \alpha_{3}, \sum_{i+1}^{3} \alpha_{i} \neq 0$, the two fixed lines of the involution on the pencil of lines with centre $e_{4}$ are

$$
\ell_{1}:\left(-\alpha_{1} \alpha_{2} \alpha_{3}-\alpha_{2} \sqrt{D}\right) z_{2}-\left(-\alpha_{1} \alpha_{2} \alpha_{3}+\alpha_{3} \sqrt{D}\right) z_{3}=0
$$

and

$$
\ell_{2}:\left(-\alpha_{1} \alpha_{2} \alpha_{3}+\alpha_{2} \sqrt{D}\right) z_{2}-\left(-\alpha_{1} \alpha_{2} \alpha_{3}-\alpha_{3} \sqrt{D}\right) z_{3}=0
$$

with $D=-\alpha_{1} \alpha_{2} \alpha_{3}\left(\alpha_{1}+\alpha_{2}+\alpha_{3}\right) \neq 0$. One only has to check that the ideal $\left(\sum_{i=1}^{3} \alpha_{i} z_{i}, \ell_{1} \cdot \ell_{2}\right)$ coincides with the ideal of $s_{\pi}$.

If $D_{\pi}$ is degenerate of the second kind with say $e_{3}, e_{4} \in \pi$ one easily checks that $\left(\alpha_{1} z_{1}+\alpha_{2} z_{2}, \alpha_{1} z_{1}^{2}+\alpha_{2} z_{2}^{2}\right)$ is the ideal of the double line $s_{\pi}=2 \overline{e_{3} e_{4}}$. Finally if $D_{\pi}$ is 
degenerate of the third kind with say $e_{1}, e_{2}, e_{3} \in \pi$ then $\left(\alpha_{1} z_{1}, \alpha_{1} z_{1}^{2}\right)$ is the ideal of the whole plane $\pi$.

In order to investigate a possible extension of the map $\Phi: M_{D} \longrightarrow \mathcal{M}_{6}^{b}$, recall its definition. Consider a Desargues configuration with von Staudt conic $s_{\pi}$. The pencil of conics passing through a complete quadrangle of $D_{\pi}$ restricts to a pencil of quartic divisors in $s_{\pi}$ whose Jacobian is a stable sextic $J$ independent of the choice of complete quadrangle and we defined $\Phi\left(D_{\pi}\right)=J$. Note that $J$ was given by $\sum_{i=1}^{4} \alpha_{i} z_{i}=\sum_{i=1}^{4} \alpha_{i} z_{i}^{2}=$ $\sum_{i=1}^{4} \alpha_{i} z_{i}^{3}=0$, if $\pi: \sum_{i=1}^{4} \alpha_{i} z_{i}=0$. Now if $D_{\pi}$ is degenerate of the first kind (respectively second) kind, $D_{\pi}$ admits still 4 (respectively 3) complete quadrangles. Restricting the pencil of conics passing through one of them to the von Staudt conic $s_{\pi}$, its Jacobian is still a sextic divisor on $s_{\pi}$. The same proof as for Theorem 3.1 also gives in this situation that the sextic divisor on $s_{\pi}$ is independent of the choice of the complete quadrangle and given by the equations

$$
\sum_{i=1}^{4} \alpha_{i} z_{i}=\sum_{i=1}^{4} \alpha_{i} z_{i}^{2}=\sum_{i=1}^{4} \alpha_{i} z_{i}^{3}=0
$$

if $\pi: \sum_{i=1}^{4} \alpha_{i} z_{i}=0$. (Notice that the difference to the above situation is that one (or two) of the constants $\alpha_{1}, \ldots, \alpha_{4}, \alpha_{1}+\ldots+\alpha_{4}$ vanishes.) Now if $D_{\pi}$ is of the first kind, the von Staudt conic is the union of two lines which intersect in a point, say $p_{0} \in \pi$ and it is easy to check that the divisor $j_{\pi}$ on $s_{\pi}$ given by equations (6) is $6 p_{0}$. If $D_{\pi}$ is of the second kind, the von Staudt conic is a double line $s_{\pi}=2 \ell_{\pi}$ and it is easy to see that the zero set of (6) is the whole line $\ell_{\pi}$. In the first case $j_{\pi}$ can be interpreted as the sextic with a 6 -fold point and in the second case not a sextic at all. In any case $j_{\pi}$ cannot (at least in an obvious way) be interpreted as a semistable binary sextic. This means that the geometric definition of the map $\Phi$ does not extend to degenerate Desargues configurations.

Remark 7.2. At first sight this seems to contradict the fact that the rational map $\Phi$ : $\bar{M}_{D} \cdots \longrightarrow \mathcal{M}_{6}^{b}$ is a morphism outside a subvariety of codimension $\geq 2$. The explanation comes from the fact that the space of all binary sextics is not separated.

\section{References}

[1] D. Avritzer and H. Lange, Pencils of Quadrics, Binary Forms and Hyperelliptic Curves, Comm. in Alg. Vol.28, N.12, p. 5541-5562, (2000).

[2] W. Blaschke, Projektive Geometrie, Birkhäuser, 3. Auflage (1954)

[3] E. Casas-Alvero and S. Xambó, Enumerative theory of conics after Halphen, LNM 1196, Springer,(1986).

[4] A. Cayley, Sur quelques théoremes de la geometrie de position, Journal für die Reine und Angewandte Mathematik (Crelle), Tome XXXL (1846), p.213-227.

[5] K. Mayer, Die Deasarguesche Konfiguration, Deutsche Math. 4, p.591-641, (1939).

[6] J. Harris, Algebraic Geometry, Springer, (1998). 
[7] D. Mumford, and J. Fogarty, Geometric Invariant Theory, Springer, (1982).

[8] Th. Reye, Geometrie der Lage, 3.Aufl. 2.Abt., Leipzig, 1886.

[9] J.G. Semple and G.T. Kneebone, Algebraic Projective Geometry, Oxford University Press (1952).

[10] Ch. von Staudt, Geometrie der Lage, Nürnberg, (1847).

[11] C. Stephanos, Mémoires sur les faisceaux de formes binaires ayant une même Jacobienne, Mémoires de l'academie des Sciences de l'Institut National de France 27 , Nr.7, (1883).

[12] J.A. Todd, Projective and Analytical Geometry, Oxford University Press, (1952).

Departamento de Matemática, UFMG

Belo Horizonte, MG 30161-970, Brasil.

dan@mat.ufmg.br
Mathematisches Institut

Bismarckstr. $1 \frac{1}{2}, 91054$, Erlangen

lange@mi.uni-erlangen.de 
This figure "Desar1.jpg" is available in "jpg" format from: http://arxiv.org/ps/math/0109151v1 
This figure "desar2.jpg" is available in "jpg" format from: http://arxiv.org/ps/math/0109151v1 
This figure "desar3.jpg" is available in "jpg" format from: http://arxiv.org/ps/math/0109151v1 
This figure "desar4.jpg" is available in "jpg" format from: http://arxiv.org/ps/math/0109151v1 